\title{
GRZEGORCZYK POINTS AND FILTERS IN BOOLEAN CONTACT ALGEBRAS
}

\author{
RAFAŁ GRUSZCZYŃSKID and ANDRZEJ PIETRUSZCZAK (1) \\ Nicolaus Copernicus University in Toruń
}

\begin{abstract}
The purpose of this paper is to compare the notion of a Grzegorczyk point introduced in [19] (and thoroughly investigated in [3, 14, 16, 18]) to the standard notions of a filter in Boolean algebras and round filter in Boolean contact algebras. In particular, we compare Grzegorczyk points to filters and ultrafilters of atomic and atomless algebras. We also prove how a certain extra axiom influences topological spaces for Grzegorczyk contact algebras. Last but not least, we do not refrain from a philosophical interpretation of the results from the paper.
\end{abstract}

\$1. Introduction. In [16, 18] we carried out an extensive analysis of one of the first systems of point-free topology by Grzegorczyk [19], based on the notion of separation (the dual notion of contact). The two papers witnessed the formulation of representation and duality theorems for a subclass of so-called Grzegorczyk structures. In particular, we established an object duality between a class of Grzegorczyk structures that satisfy the countable chain condition and the concentric spaces (a subclass of the class of regular spaces), which satisfy the topological version of the same condition.

A particular idiosyncrasy of Grzegorczyk's approach to point-free topology is his definition of a point, which is a formal reflection of the geometrical intuition of a point as a system of "shrinking" regions of space. We have devoted much attention to elucidating the idea in [14-16, 18], so we refer the reader to these works if they want to familiarize themselves with an informal (yet precise) analysis of the notion.

One of the problems that occupied us in the aforementioned works was the relation of Grzegorczyk points to other classical point-like constructions, such as ultrafilters and maximal round filters. We have only scratched the surface of the problem, yet we demonstrated that the following two second-order sentences:

(a) Every Grzegorczyk point is an ultrafilter, and

(b) Every ultrafilter is a Grzegorczyk point

correspond to the following first-order and second-order sentences, respectively:

$\left(\mathrm{a}^{\prime}\right)$ Each region is separated from its complement.

(cf. Theorem 8.4)

$\left(\mathrm{b}^{\prime}\right)$ The structure is finite.

(cf. Theorem 9.1)

Received: April 25, 2020.

2020 Mathematics Subject Classification: Primary 06E25, Secondary 03G05, 06E15

Key words and phrases: Boolean contact algebras, Grzegorczyk contact algebras, Grzegorczyk points, round filters, ultrafilters, Fréchet filter, region-based topology, point-free topology, compactifications, concentric topological spaces, lob-spaces.

(C) The Author(s), 2021. Published by Cambridge University Press on behalf of Association for Symbolic Logic. This is an Open Access article, distributed under the terms of the Creative Commons Attribution licence (https://creativecommons.org/licenses/by/4.0/), which permits unrestricted re-use, distribution, and reproduction in any medium, provided the original work is properly cited. 
We proved as well that every Grzegorczyk point is a maximal round filter. For the sake of completeness of the presentation, some of these results are contained in this paper.

Here, we aim to examine how Grzegorczyk points behave and relate to filters and ultrafilters in atomless and atomic Boolean contact algebras, which in a very natural way relate to Grzegorczyk structures from the earlier works. To be more precise, previously we worked with mereological fields, which are structures that always lack the zero element and might lack the unity. In all other respects, they bear a very strong resemblance to Boolean algebras, thanks to the classical theorems to which we refer in Sections 6 and 7 of [16]. Thanks to these theorems, all results obtained in the earlier works are easily translatable into analogous results in the framework of Boolean algebras. We have also replaced the primitive binary relation of separation with its dual, the contact relation. The main reason to do so was the fact that since more or less the beginning of the twenty-first century and the seminal works of Stell [23], Düntsch \& Winter [10, 11] and Dimov \& Vakarelov [8, 9], Boolean contact algebras have been the standard framework for doing region-based topology. Thus, with technical results in focus and philosophical considerations aside, contact algebras are the most natural choice for the presentation of advances in the study of Grzegorczyk point-free topology.

§2. Motivations. There are various reasons for doing region-based theories of space. Probably the main one is to describe spatial relations in terms of concepts that can be rooted in the sensory experience. Such were motivations for both Region Connection Calculus of Cohn et al. [5] and Boolean contact algebras. From the purely mathematical point of view, algebraic parts of these theories focused on finding suitable topological representations and duality theorems, most notably via the spaces of clusters as, among others, in the works of Düntsch \& Winter [11] and Dimov \& Vakarelov [8]. In closely related De Vries algebras from [7] maximal round filters play a crucial role in algebraization of the topological notion of compactness.

The approach taken by Grzegorczyk [19] seems to have yet another motivationfind spatially satisfactory construction of points in terms of regions and separation (equivalently: contact) relation. Grzegorczyk points are, in a way, generalizations of Tarski's points as sets of concentric balls from his geometry of solids [25], and they embody the idea of a point as a system of shrinking regions of space.

From both philosophical and technical points of view, it is interesting (at least for us, as the authors of the paper) how second-order conditions expressing dependencies between various constructions of points relate to either first- or second-order properties of Boolean contact algebras. This line of research was initiated by Biacino \& Gerla [3] with finding sufficient conditions for Grzegorczyk points and Whitehead points from [26] to coincide. We believe that further investigation of dependencies between various constructions of points can bring interesting results in region-based theories of space, even more so because it is very little examined fragment of such theories.

As pointed to in the introduction, in our previous works we showed certain correspondences between statements about points of contact algebras and their properties. In particular, we found it interesting how Grzegorczyk points relate to the standard filter constructions of Boolean contact algebras. Among these, the Fréchet filter plays a distinguished role in the class of atomic Boolean algebras, and, interestingly, its relation to Grzegorczyk points is independent from the axioms of 
Grzegorczyk contact algebras. We venture to say that this may be interpreted as the fact that the notion of Fréchet filter is independent from the spatial intuitions behind the definition of Grzegorczyk points. Investigating consequences of the statement "Fréchet filter is a Grzegorczyk point" may be interpreted philosophically as answering the question what are consequences of forcing our spatial intuitions about points into the notion of Fréchet filter? To us as the authors, logicians with a strong predilection toward philosophical aspects of mathematical theories, it seemed a very stimulating question which is faced and solved in the paper.

It is hard to say now what, if any, fruits bears the theory of correspondences between statements about points of Boolean contact algebras and their familiar properties. However, in our opinion, it is a path that is worth studying as it may lead to new perspectives on various - both mathematical and philosophical - aspects of regionbased theories of space.

§3. Boolean quasi-contact algebras. This section is a refresher of the standard notions, axioms and elementary properties of contact algebras that are essential for the key results of the paper.

A Boolean quasi-contact algebra (BQCA for short) is any pair $\mathfrak{R}=\langle\mathfrak{B}, \mathrm{C}\rangle$, where $\mathfrak{B}=\langle R, \sqcap, \sqcup,-, 0,1\rangle$ is a non-degenerate Boolean algebra ${ }^{1}$ (BA for short) and $\mathrm{C}$ is a binary contact relation on $R$ which meets the following four axioms: ${ }^{2}$

$$
\begin{gathered}
\forall_{x \in R} 0 \text { \& } x, \\
\forall_{x, y \in R}(x \subset y \Longrightarrow y \subset x), \\
\forall_{x \in R^{+}} x \text { C } x, \\
\forall x, y \in R\left(x \leq y \Longrightarrow \forall_{z \in R}(z \subset x \Rightarrow z \subset y)\right),
\end{gathered}
$$

where $\varnothing$ is the complement of $C, R^{+}:=R \backslash\{0\}$ and $\leq$ is the standard partial order defined by:

$$
x \leq y: \Longleftrightarrow x \sqcap y=x \Longleftrightarrow x \sqcup y=y .
$$

Furthermore, we will use the standard strict partial order defined by:

$$
x \leq y: \Longleftrightarrow x \leq y \wedge x \neq y \Longleftrightarrow x \leq y \wedge y \not \leq x .
$$

All elements of the domain $R$ will be called regions; the region 0 will be called empty; and all regions from $R^{+}$will be called non-empty. In the case $x \subset y$ (resp. $x \mathbb{C} y ; x \leq y$; $x \leq y$ ) we will say that $x$ is in contact with $y$ (resp. $x$ is separated from $y$; $x$ is part of $y$; $x$ is a proper part of $y)$. Axioms $(\mathrm{C} 0)-(\mathrm{C} 3)$ say, respectively: 0 is separated from any region; $\mathrm{C}$ is symmetric and reflexive on $R^{+}$; and if one region is part of another, then each region in contact with the first is also in contact with the other. In BAs condition (C3) is equivalent to

$$
\forall_{x, y, z \in R}(z \subset x \vee z \subset y \Longrightarrow z \subset x \sqcup y) .
$$

1 We use [22] as the standard reference for concepts related to and results about Boolean algebras.

2 What we call quasi-contact is the weak contact in the sense of [12]. Our axioms coincide with those chosen by the authors of the aforementioned paper. 
From (C3) and the reflexivity of $\leq$ we obtain that in place of $(\mathrm{C} 2)$ we can assume that $C$ includes $\leq$ limited to non-empty regions:

$$
\forall_{x, y \in R^{+}}(x \leq y \Longrightarrow x \text { C } y) . \quad\left(\leq\left.\right|_{\mathrm{R}^{+}} \subseteq \mathrm{C}\right)
$$

So every non-empty region is in contact with 1 :

$$
\forall x \in R^{+} x \mathrm{C} 1 \text {. }
$$

We will also use two auxiliary binary relations on $R: \bigcirc$ and $\perp$. We define the first by:

$$
x \circ y: \Longleftrightarrow x \sqcap y \neq 0 \Longleftrightarrow \exists_{z \in R^{+}}(z \leq x \wedge z \leq y),
$$

and we let the second be the complement of $\bigcirc$. In the case $x \circ y$ (resp. $x \perp y$ ) we will say that $x$ overlaps $y$ (resp. $x$ is disjoint from $y$ ). From $(\mathrm{C} 3),(\mathrm{C} 1)$ and $\left(\leq\left.\right|_{\mathrm{R}^{+}} \subseteq \mathrm{C}\right.$ ) we have:

$$
\begin{gathered}
\forall_{x, y \in R}\left(x \mathrm{C}-y \Longleftrightarrow \exists_{z \in R}(x \mathrm{C} z \wedge z \perp y)\right), \\
\forall_{x, y \in R}(x \mathbb{C}-y \Longrightarrow x \leq y), \\
\forall_{x, y \in R}(x \circ y \Longrightarrow x \text { С } y) .
\end{gathered}
$$

Yet another auxiliary standard relation of non-tangential inclusion is defined by:

$$
x \ll y: \Longleftrightarrow x \varnothing-y .
$$

In the case $x \ll y$ we say that $x$ is a non-tangential part of $y$. From (3.3) it follows that:

$$
x \ll y \Longleftrightarrow \forall_{z \in R}(z \mathrm{C} x \Rightarrow z \circ y) .
$$

From $(\mathrm{C} 0),(\mathrm{C} 1),\left(\leq\left.\right|_{\mathrm{R}^{+}} \subseteq \mathrm{C}\right),\left(\mathrm{df}^{\prime} \ll\right)$ we obtain that every region is a non-tangential part of 1 and each non-tangential part of a given region is its (ordinary) part:

$$
\begin{gathered}
\forall_{x \in R} x \ll 1, \\
\forall_{x, y \in R}(x \ll y \Longrightarrow x \leq y) .
\end{gathered}
$$

From $\left(\mathrm{df}^{\prime} \ll\right),(\mathrm{df} \bigcirc)$ and the transitivity of $\leq$ we have:

$$
\forall_{x, y, u, v \in R}(u \leq x \wedge v \ll y \wedge u \subset v \Longrightarrow x \circ y) .
$$

Furthermore, we have the following property of $\ll$ :

$$
\forall_{x, y, z, u \in R}(x \leq u \wedge u \ll y \wedge y \leq z \Rightarrow x \ll z) .
$$

It — together with $(\ll \subseteq \leq)$ and the reflexivity of $\leq$ - gives the transitivity of $\ll$ :

$$
\forall_{x, y, z \in R}(x \ll y \wedge y \ll z \Longrightarrow x \ll z) \text {. }
$$

We will use three families of filters in BAs. The first one will be the family Ult of all ultrafilters, i.e., proper maximal filters. The second one will be the family of principal filters of the form $\uparrow x:=\{u \in R \mid x \leq u\}$ for an $x \in R$ (the set $\uparrow x$ is called a principal filter generated by $x$ ). Let $\mathbf{P F}$ and $\mathbf{P F}_{\mathrm{At}}$ be families of all principal filters and of principal filters generated by atoms, respectively. The third family will be the family of free filters which satisfy the following condition: there is no $x \in R$ such that for any $y \in F$ we have $x \leq y$. Every ultrafilter is free or belongs to $\mathbf{P F}_{\mathrm{At}}$. 
Moreover, two families of filters in BQCAs will play special roles in the sequel. The first one will be the family Rnd of so-called round (or contracting) filters which are proper filters satisfying:

- for any $x \in F$ there is a $y \in F$ such that $y \ll x$.

From $(\circ \subseteq \mathrm{C}),\left(\mathrm{df}^{\prime} \ll\right)$ and $(\ll \subseteq \leq)$ we obtain:

Lemma 3.1. For any round filter F we have: $\forall_{x \in R}\left(\forall_{z \in F} z \mathrm{C} x \Longleftrightarrow \forall_{z \in F} z \circ x\right)$.

The second one is the family M.Rnd of all proper maximal filters in the family Rnd. ${ }^{3}$

LEMma 3.2 [18, lemma 10.6]. If a round filter F satisfies the condition $\forall_{x, y \in R}\left(\left(\forall_{z \in F} z \mathrm{C}\right.\right.$ $x \wedge x \ll y) \Longrightarrow y \in F$ ), then F belongs to M.Rnd.

It is easy to check that the structures $\langle\mathfrak{B}, O\rangle$ and $\left\langle\mathfrak{B}, R^{+} \times R^{+}\right\rangle$are BQCAs. We will call them trivial BQCAs. The relation $\bigcirc$ is the smallest contact relation on $\mathfrak{B}$ (cf. [10, proposition 2.2(1)]. From $\left(\mathrm{df}^{\prime} \ll\right),(\bigcirc \subseteq \mathrm{C})$ and $(\mathrm{C} 2)$ we obtain:

$$
\mathrm{C}=\mathrm{O} \Longleftrightarrow \ll=\leq \Longleftrightarrow \ll \text { is reflexive. }
$$

Hence all filters in $\langle\mathfrak{B}, \bigcirc\rangle$ are round; and so Ult $=$ M.Rnd.

§4. Boolean contact algebras. After [10], we will call a Boolean contact algebra (BCA for short) any BQCA satisfying

$$
\begin{gathered}
\forall_{x, y, z \in R}(z \mathrm{C} x \sqcup y \Longrightarrow z \mathrm{C} x \vee z \subset y), \\
\forall_{x \in R \backslash\{1\}} \exists_{y \in R^{+}} x \text { ८ } y .
\end{gathered}
$$

From (3.1) and (C4) we have:

$$
\forall x, y, z \in R(z \mathrm{C} x \sqcup y \Longleftrightarrow z \mathrm{C} x \vee z \mathrm{C} y) \text {. }
$$

By means of $(3.5),(\mathrm{df} \ll),(\mathrm{C} 3),(\ll \subseteq \leq),\left(\leq\left.\right|_{\mathrm{R}^{+}} \subseteq \mathrm{C}\right),(\mathrm{C} 1),(3.2)$ and the antisymmetry of $\leq$, we can prove:

LEMMA 4.1. In BQCAs condition (C5) is equivalent to each of the following:

$$
\begin{gathered}
\forall_{x \in R^{+}} \exists_{y \in R^{+}} y \ll x, \\
\forall_{x, y \in R}\left(\forall_{z \in R}(z \mathrm{C} x \Rightarrow z \mathrm{C} y) \Longrightarrow x \leq y\right), \\
\forall_{x, y \in R}\left(x \leq y \Longleftrightarrow \forall_{z \in R}(z \mathrm{C} x \Rightarrow z \mathrm{C} y)\right), \\
\forall_{x, y \in R}\left(\forall_{z \in R}(z \mathrm{C} x \Leftrightarrow z \mathrm{C} y) \Longrightarrow x=y\right) .
\end{gathered}
$$

REMARK 4.2. Dimov \& Vakarelov [8, p. 213] analyzed contact algebras as structures of the form $\langle\mathfrak{B}, \mathrm{C}\rangle$ satisfying $(\mathrm{C} 0)-(\mathrm{C} 2)$ and (4.1). The algebras that additionally satisfy (extc) are named by them as extensional contact algebras (p. 215). In Lemma 2.2 of [8] the authors demonstrate that conditions $\left(\operatorname{ext}_{\mathrm{C}}\right),(\mathrm{C} 5),\left(\exists_{\ll}\right)$ and $\left(\mathrm{C} 3_{\mathrm{c}}\right)$ are equivalent in any contact algebra. But, as the above lemma shows, BQCAs are enough for this, i.e., the condition $(\mathrm{C} 4)$ can be omitted in the proofs.

${ }^{3}$ Generally, Rnd $\cap$ Ult $\subseteq$ M.Rnd $\subseteq$ Rnd. But there are BCAs in which M.Rnd $\nsubseteq$ Rnd $\cap$ Ult. In Section 6 we will introduce the sixth family of filters, so-called Grzegorczyk points. 
Düntsch \& Winter [11] treated Boolean contact algebras as structures of the form $\langle\mathfrak{B}, \mathrm{C}\rangle$ satisfying $(\mathrm{C} 0)-(\mathrm{C} 4)$ and $\left(\mathrm{C}_{\mathrm{c}}\right)$. They showed that these conditions entail $(\mathrm{C} 5)$ and (3.1) (Lemma 3.2).

It is easy to check that trivial BQCAs of the form $\langle\mathfrak{B}, 0\rangle$ (see p. 21) are BCAs. In the sequel we will use ' $\mathrm{BCA}^{\mathrm{O}}$ ' as a general name for all $\mathrm{BCAs}$ with $\mathrm{C}=0$. The BQCAs of the form $\left\langle\mathfrak{B}, R^{+} \times R^{+}\right\rangle$are BCAs iff $R^{+}=\{1\}$ iff $\bigcirc=\leq$ iff $R^{+} \times R^{+}=\bigcirc$. So all trivial examples of BCAs are $\mathrm{BCA}^{\circ} \mathrm{s}$.

Let At be the set of atoms of a given BA. Directly from $\left(\exists_{\ll}\right)$ and $(\ll \subseteq \leq)$ we have:

$$
\forall a \in \text { At } a \ll a .
$$

Hence, using $(\circ \subseteq \mathrm{C}),\left(\mathrm{df}^{\prime} \ll\right)$ and (3.7), we obtain:

$$
\forall a \in \operatorname{At} \forall_{x \in R}(a \subset x \Longleftrightarrow a \circ x \Longleftrightarrow a \leq x \Longleftrightarrow a \ll x) \text {. }
$$

From this and $\left(\mathrm{df}^{\prime} \ll\right),(\mathrm{C} 1)$, and (4.1), we get a generalization of (4.2):

$$
\forall a_{1}, \ldots, a_{n} \in \mathrm{At} a_{1} \sqcup \cdots \sqcup a_{n} \ll a_{1} \sqcup \cdots \sqcup a_{n} .
$$

For any $x \in R$ we put: $\mathrm{At}_{x}:=\{a \in \mathrm{At} \mid a \leq x\}$ and $\mathrm{At}_{x}^{\perp}:=\{a \in \mathrm{At} \mid a \perp x\}$. It is clear that the atomicity of a given BA implies that $x=\sup \mathrm{At}_{x}$. A BQCA is atomic iff it is based on an atomic BA. The following fact will be used in the proof of Theorem 7.8 .

Lemma 4.3. In any atomic BCA we have:

$$
\forall x, y \in R\left(x \subset y \wedge x \perp y \Longrightarrow \text { both } \mathrm{At}_{x} \text { and } \mathrm{At}_{y} \text { are infinite }\right) .
$$

Proof. Assume for a contradiction that $x \mathrm{C} y, x \perp y$ and $\mathrm{At}_{x}$ is finite. Then for some atoms $a_{1}, \ldots, a_{n}$ we have $x=a_{1} \sqcup \cdots \sqcup a_{n}$. So, by (C1), (4.1) and (4.3), we have: $x \subset y$ iff $a_{1} \subset y$ or $\ldots$ or $a_{n} \subset y$ iff $a_{1} \leq y$ or $\ldots$ or $a_{n} \leq y$. Therefore $x \circ y$, a contradiction.

We say that a BA is finite-cofinite iff for any $x \in R$ either $x \in\{0,1\}$ or there are $n>0$ and $a_{1}, \ldots, a_{n} \in$ At such that either $x=a_{1} \sqcup \cdots \sqcup a_{n}$ or $x=-\left(a_{1} \sqcup \cdots \sqcup a_{n}\right)$. Clearly, all finite BAs are finite-cofinite. Düntsch \& Winter [10] showed that the only BCAs that can be obtained from finite-cofinite BAs are the trivial $\mathrm{BCAs}^{\circ}$.

Proposition 4.4 [10, proposition 2.2(2)]. If $\mathfrak{B}$ is finite-cofinite, then $\langle\mathfrak{B}, \bigcirc\rangle$ is the only $B C A$ based on $\mathfrak{B}$.

Notice that every non-empty region which is not an atom has at least two nontangential non-empty proper parts which are separated from each other:

$$
\forall_{x \in R^{+} \backslash A \mathrm{t}} \exists_{u, v \in R^{+}}(u \ll x \wedge v \ll x \wedge u \ell v \wedge u \neq x \neq v) .
$$

Indeed, assume that $x \in R^{+} \backslash$ At. Then there are $y, z \in R^{+}$such that $y \perp z$ and $x=$ $y \sqcup z$. Hence, by $\left(\exists_{\ll}\right)$, there are $u, v \in R^{+}$such that $u \ll y \lesseqgtr x$ and $v \ll z \lessgtr x$. So, by (3.6) and ( $\ll \leq \leq)$, we have $u \mathbb{\ell} v$. Moreover, by (3.7), we have $u \ll x, v \ll x$ and $u \neq x \neq v$.

A BQCA is atomless iff it is based on an atomless BA.

Remark 4.5. (i) Dimov \& Vakarelov [8, definition 3.3] define another type of filters. Namely, a filter $F$ is an end iff $F$ is a round filter satisfying the following condition:

$$
\forall x, y \in R(x \not) y-x \in F \vee-y \in F) \text {. }
$$


They show (Lemma 3.2(viii)) that all end filters belong to M.Rnd. Moreover, they prove (Proposition 3.1(iii)) that in any BCA satisfying the so-called interpolation axiom

$$
\forall x, y \in R\left(x \ll y \Longrightarrow \exists_{z \in R} x \ll z \ll y\right),
$$

every filter from M.Rnd is an end.

(ii) Gruszczyński [14] proves that in any BCA satisfying (IA), a filter $F$ belongs to M.Rnd if and only if $F$ satisfies the following condition:

$$
\forall_{x, y \in R}\left(\forall_{z \in F}(x \mathrm{C} z \wedge y \mathrm{C} z) \Longrightarrow x \mathrm{C} y\right) \text {. }
$$

The following lemma will be used to obtain Proposition 4.8.

LEMMA 4.6. In any atomless BCA, any filter F satisfying the following condition is free:

$$
\forall_{x, y \in R^{+}}\left(\forall_{z \in F}(x \leq z \wedge y \leq z) \Longrightarrow x \text { C } y\right) .
$$

Proposition 4.7. In any atomless $B C A$, if $F \in \mathbf{U l t} \cup$ M.Rnd then $F$ satisfies the following condition:

$$
\forall_{x, y \in R^{+}}\left(\forall_{z \in F}(x \leq z \wedge y \leq z) \Longrightarrow x \circ y\right) .
$$

Proof. Suppose that in an atomless BCA for a filter $F$ there are $x, y \in R^{+}$such that (a) $x \perp y$ and (b) for any $z \in F: x \leq z$ and $y \leq z$. Then, by (a), either $x$ or $y$ does not belong to $F$. Assume the first possibility holds: (c) $x \notin F$. By (4.5), for some $u_{1} \in R^{+}$ we have $u_{1} \ll x$ and $u_{1} \neq x$. Observe that by (b) and (3.7), $u_{1}$ is a non-tangential part of any $z \in F$. By means of (4.5) and the axiom of dependent choices we produce a sequence $\left\langle u_{n} \mid n<\omega\right\rangle$ such that $u_{0}:=x, u_{i+1} \ll u_{i}$ and $u_{i+1} \neq u_{i}$. The filter $\uparrow X$ generated by the set of the terms of the sequence is obviously round, and by (b) and (c) it is the case that $F \subsetneq \uparrow X$. Therefore, $F \notin$ Ult and $F \notin$ M.Rnd.

Finally, from Lemma 4.6, Proposition 4.7 and $(\bigcirc \subseteq C)$ we obtain:

Proposition 4.8. In any atomless BCA, all filters from M.Rnd are free. ${ }^{4}$

§5. Pre-points. Let $\mathfrak{R}$ be a BQCA based on a BA $\mathfrak{B}$. A pre-point of $\mathfrak{R}$ is any non-empty set $Q$ of non-empty regions which satisfies the following conditions:

$$
\begin{gathered}
\forall_{u, v \in Q}(u=v \vee u \ll v \vee v \ll u), \\
\forall_{u \in Q} \exists_{v \in Q} v \ll u, \\
\forall_{x, y \in R}\left(\forall_{u \in Q}(u \circ x \wedge u \circ y) \Longrightarrow x \subset y\right) .
\end{gathered}
$$

The purpose of this definition is to formally grasp the intuition that a point is a system of diminishing regions determining a unique location in space. We call it a pre-point, since if we understand a point as a perfect representation of some location in space, then it may happen that two different sets of regions represent one and the same location. Further, we will identify such pre-points to act as one point. Let $\mathbf{Q}$ be the set of all pre-points of $\mathfrak{R}$.

4 In the primary version of the paper we proved this proposition only for atomless BCAs satisfying (IA). However, one of the reviewers suggested to us that assumption (IA) is redundant, which prompted us to look for Proposition 4.7. 
Notice that by $(\ll \subseteq \leq)$, all pre-points are chains with respect to the part of relation. Furthermore, by $(\mathrm{r} 1),(\ll \subseteq \leq)$ and $(\mathrm{df} \leq)$, all pre-points have the finite intersection property. So each pre-point $Q$ generates the filter $\uparrow Q:=\left\{x \in R \mid \exists_{u_{1}, \ldots, u_{n} \in Q} u_{1} \sqcap \cdots \sqcap\right.$ $\left.u_{n} \leq x\right\}$. From (r1) we have $\uparrow Q=\left\{x \in R \mid \exists_{u \in Q} u \leq x\right\}$.

By (4.2) and $(O \subseteq C)$, all singletons composed of atoms are pre-points in all BCAs:

Lemma 5.1. In any BCA, for any $a \in$ At the singleton $\{a\}$ is a pre-point.

\section{§6. Grzegorczyk points.}

6.1. Grzegorczyk points of BQCAs. Let $\mathfrak{R}$ be a BQCA based on a BA $\mathfrak{B}$. By a Grzegorczyk point (G-point for short) of $\mathfrak{R}$ we will mean any filter generated by a pre-point of $\mathfrak{R}$. Thus, for any filter $F$ we have:

a filter $F$ is a G-point iff there is a $Q \in \mathbf{Q}$ such that $F=\uparrow Q$.

Let Gpt be the set of all G-points of $\mathfrak{R}$. Its elements will be denoted by small gothic letters ' $\mathfrak{p}$ ' and ' $q$ '.

Every G-point is a maximal filter in Gpt (see [16]):

$$
\forall_{\mathfrak{p}, \mathfrak{q} \in \mathbf{G p t}}(\mathfrak{p} \subseteq \mathfrak{q} \Longrightarrow \mathfrak{p}=\mathfrak{q})
$$

Furthermore, in [18] we proved that in all BQCAs:

$$
\begin{aligned}
& \forall_{x, y \in R} \forall_{\mathfrak{p} \in \mathbf{G p t}}\left(\forall_{z \in \mathfrak{p}}(x \circ z \wedge y \circ z) \Longrightarrow x \subset y\right), \\
& \forall_{x, y \in R}\left(x \ll y \Longrightarrow \forall_{\mathfrak{p} \in \mathbf{G p t}}\left(y \in \mathfrak{p} \vee \exists_{z \in \mathfrak{p}} z \perp x\right)\right) .
\end{aligned}
$$

For Grzegorczyk contact algebras, which are presented in Section 8 and which are BCAs, we obtain the converse implications (8.2) and (8.3).

In the light of (r2) and (3.7) we have Gpt $\subseteq$ Rnd. Hence, by (6.2) and Lemmas 3.1 and 3.2, for any BQCA we obtain the following fact:

\section{Proposition 6.1. Every G-point in a BQCA belongs to M.Rnd. ${ }^{5}$}

Hence, in the light of Lemma 3.1, condition (6.1) is equivalent to the following (cf. condition $(\%))$ :

$$
\forall_{\mathfrak{p} \in \mathbf{G p t}} \forall_{x, y \in R}\left(\forall_{z \in \mathfrak{p}}(x \mathrm{C} z \wedge y \mathrm{C} z) \Longrightarrow x \mathrm{C} y\right) .
$$

Now we will consider BQCAs which are complete.

Proposition 6.2. 1. In any complete BQCA, every G-point being an ultrafilter is principal and generated by an atom, i.e., $\mathbf{G p t} \cap \mathbf{U l t} \subseteq \mathbf{P F}_{\mathrm{At}}$.

2. In any atomless complete BQCA, no G-point is an ultrafilter, i.e., Gpt $\cap$ Ult $=\emptyset$.

Proof. Ad 1. Every ultrafilter is free or belongs to $\mathbf{P F}_{\mathrm{At}}$. Every G-point is generated by a chain. But no free ultrafilter in a complete BA is generated by a chain (see, e.g., [21, lemma 43]). Ad 2. Directly from point 1.

$\overline{5}$ In $[14,18]$ it was proven only for GCAs. 
6.2. G-points of BCAs. In the general case of BCAs, from Lemma 5.1 and Proposition 6.1 we obtain:

Proposition 6.3. In any BCA, every principal filter generated by an atom is a G-point being an ultrafilter and belongs to $\mathbf{M . R n d}$, i.e., $\mathbf{P F}_{\mathrm{At}} \subseteq \mathbf{G p t} \cap \mathbf{U l t} \cap \mathbf{M}$.Rnd $=\mathbf{G p t} \cap \mathbf{U l t}$.

Hence we have:

COROLLARY 6.4. In any BCA, every principal ultrafilter is a G-point generated by an atom, i.e., $\mathbf{P F} \cap \mathbf{U l t} \subseteq \mathbf{G p t} \cap \mathbf{P F}_{\mathrm{At}}$.

Moreover, by Propositions 6.2(1) and 6.3, for complete BCAs we have:

Corollary 6.5. In any complete BCA, Gpt $\cap \mathbf{U l t}=\mathbf{P F}_{\mathrm{At}}=\mathbf{G p t} \cap \mathbf{U l t} \cap \mathbf{M}$.Rnd.

From Propositions 6.1 and 6.3 for atomic BCAs we obtain:

PROPOSITION 6.6. In any atomic BCA, every G-point being a principal filter is generated by an atom, i.e., $\mathbf{G p t} \cap \mathbf{P F} \subseteq \mathbf{P F}_{\mathrm{At}}$.

Proof. By Proposition 6.1, if $\uparrow x \in \mathbf{G p t}$ then $\uparrow x \in$ M.Rnd. Moreover, for some $a \in$ At we have $a \leq x$. Hence $\uparrow x \subseteq \uparrow a$. But, $\uparrow a \in$ Gpt, by Proposition 6.3. Therefore $x=a$.

For atomless BCAs, by Propositions 6.3 and 4.8, we obtain: ${ }^{6}$

THEOREM 6.7. Every G-point of an atomless BCA is a free filter.

Finally, we consider the case of $\mathrm{BCAs}^{\circ}$. Firstly, since all filters in $\mathrm{BCAs}^{\circ}$ are round, from Proposition 6.1 we get:

Lemma 6.8. In any $B C A^{\circ}$, Gpt $\subseteq$ M.Rnd $=$ Ult.

Secondly, there are, however, $\mathrm{BCA}^{\circ} \mathrm{s}$ in which Gpt $\subsetneq$ M.Rnd:

Proposition 6.9. In any infinite complete atomic $B C A^{\circ}$, Gpt $\subsetneq$ M.Rnd $=$ Ult.

Proof. Every infinite complete atomic BA has a free ultrafilter. But, by Proposition 6.2(1), no free ultrafilter is a G-point. Hence Gpt $\subsetneq$ Ult $=$ M.Rnd, by Lemma 6.8.

From finite-cofinite BAs we only get $\mathrm{BCAs}^{\circ}$ (see Proposition 4.4). Hence from Propositions 6.3 and 6.9, Corollary 6.5 and Lemma 6.8, respectively, we get:

Corollary 6.10. 1. In any finite $B C A$, $\mathbf{G p t}=\mathbf{P F}_{\mathrm{At}}=\mathbf{U l t}=\mathbf{M} . \mathbf{R n d}$.

2. In any infinite finite-cofinite $B C A, \mathbf{P F}_{\mathrm{At}} \subseteq \mathbf{G p t} \subseteq \mathbf{U l t}=\mathbf{M} . \mathbf{R n d}$.

§7. The Fréchet filter vs. G-points. As we have seen in any BCA each principal ultrafilter is a G-point generated by an atom (cf. Corollary 6.4). In the case of finite BCAs we have $\mathbf{G p t}=\mathbf{P F}_{\mathrm{At}}=$ Ult (cf. Corollary 6.10.(1)). We will now answer the following two questions: Are there any other G-points than principal ultrafilters in infinite atomic algebras? and In which infinite atomic BCAs the Fréchet filter can or cannot be a G-point? The subclass of BCAs which will serve to answer those question corresponds to a certain subclass of GCAs.

Let $\mathfrak{B}$ be an infinite atomic BA. Then the set cAt of all coatoms has a finite intersection property and generates the so-called Fréchet (or cofinite) filter $\mathrm{F}_{\mathrm{c}}$, which is free. We will use the following properties of $F_{c}$.

$\overline{6}$ We can also use $(6.1),\left(\leq\left.\right|_{\mathrm{R}^{+}} \subseteq \mathrm{C}\right)$ and Lemma 4.6. 
Lemma 7.1. For any $x \in \mathrm{F}_{\mathrm{c}}$ there are $a_{1}, \ldots, a_{n} \in \mathrm{At}(n>0)$ such that $x=-\left(a_{1} \sqcup\right.$ $\left.\cdots \sqcup a_{n}\right)$.

From Lemma 7.1 and Proposition 4.4 we obtain:

Corollary 7.2. If $\mathrm{F}_{\mathrm{c}} \in \mathbf{U l t}$, then $\mathfrak{B}$ is finite-cofinite, and so $\langle\mathfrak{B}, \bigcirc\rangle$ is the only $B C A$ based on $\mathfrak{B}$.

The following two lemmas are analogous to the facts given in [4], where the completeness of a Boolean algebra $\mathfrak{B}$ is assumed and the fact that $\mathfrak{B}$ is isomorphic to the algebra of sets over At is used.

LeMma 7.3. Let $\mathfrak{B}$ have countably many atoms: $a_{1}, a_{2}, \ldots$. Then $\mathrm{F}_{\mathrm{c}}$ is generated by the chain $\mathrm{C}_{\mathrm{c}}:=\left\{-a_{1} \sqcap \cdots \sqcap-a_{n} \mid n \in \omega\right\}$.

Proof. If $x \in \mathrm{F}_{\mathrm{c}}$ then for some $k>0$ and $a_{i_{1}}, \ldots, a_{i_{k}} \in$ At we have $-a_{i_{1}} \sqcap \ldots \sqcap-$ $a_{i_{k}} \leq x$. Hence $\mathrm{C}_{\mathrm{c}} \ni-a_{1} \sqcap \cdots \sqcap-a_{\max \left\{i_{1}, \ldots, i_{k}\right\}} \leq-a_{i_{1}} \sqcap \cdots \sqcap-a_{i_{k}} \leq x$. So $\mathrm{F}_{\mathrm{c}} \subseteq \uparrow \mathrm{C}_{\mathrm{c}}$. Furthermore, if for some $n \in \omega$ we have $-a_{1} \sqcap \cdots \sqcap-a_{n} \leq x$, then $-x \leq a_{1} \sqcup \cdots \sqcup a_{n}$, which means that $-x$ is composed of finitely many atoms, i.e., $x \in \mathrm{F}_{\mathrm{c}}$. So also $\uparrow \mathrm{C}_{\mathrm{c}} \subseteq$ $\mathrm{F}_{\mathrm{c}}$.

The proof of the lemma below is after [4], yet omits the completeness assumption.

LEMMA 7.4. If $\mathfrak{B}$ has uncountably many atoms, then $\mathrm{F}_{\mathrm{c}}$ is not generated by a chain .

Proof. Assume for a contradiction that $\mathrm{F}_{\mathrm{c}}$ is generated by a chain $C$. Then $C \subseteq \mathrm{F}_{\mathrm{c}}$. Hence, by Lemma 7.1, for any $x \in C$ there are $n>0$ and $a_{1}, \ldots, a_{n} \in$ At such that $-x=a_{1} \sqcup \cdots \sqcup a_{n}$. Therefore the set $\mathrm{At}_{x}^{\perp}$ is finite.

Furthermore, since $C$ is a chain, for all $x, y \in C$ such that $x \neq y$ either $\mathrm{At}_{x}^{\perp} \subsetneq \mathrm{At}_{y}^{\perp}$ or $\mathrm{At}_{y}^{\perp} \subsetneq \mathrm{At}_{x}^{\perp}$. Thus, the function $C \ni x \mapsto \operatorname{Card}\left(\mathrm{At}_{x}^{\perp}\right) \in \omega$ is injective; and so $C$ and $\bigcup_{x \in C} \mathrm{At}_{x}^{\perp}$ are countable. Hence for some $a_{0} \in$ At we have $a_{0} \notin \bigcup_{x \in C} \mathrm{At}_{x}^{\perp}$, i.e., for any $x \in C$ we have $x \not \leq-a_{0}$. However, for all $a \in \mathrm{At}$ it is the case that $-a \in \mathrm{F}_{\mathrm{c}}$. Therefore, since $\mathrm{F}_{\mathrm{c}}$ is generated by $C$, for some $x_{a} \in C$ we have $x_{a} \leq-a$; a contradiction.

Furthermore, let us remind that:

LEMMA 7.5. $\mathrm{F}_{\mathrm{c}}$ is included in any free filter in $\mathfrak{B}$.

Now we will show two classes of BCAs in which $F_{c}$ is not a G-point. From them we will also get two classes of GCAs in which $F_{c} \notin \mathbf{G p t}$ (see Section 10). Keep in mind that whether $F_{c}$ is generated by the chain depends on whether $\mathfrak{B}$ has countably many atoms.

Because all G-points are generated by chains, in the light of Lemma 7.4 we obtain:

Proposition 7.6. There is no atomic BQCA with uncountably many atoms such that:

$$
\mathrm{F}_{\mathrm{c}} \in \mathbf{G p t} \text {. }
$$

Moreover, we have the following generalization of model 6.18 from [14]:

Proposition 7.7. There is no infinite complete atomic $B C A^{\circ}$ that satisfies $\left(\mathrm{pF}_{\mathrm{c}}\right)$.

Proof. Let $\mathfrak{R}=\langle\mathfrak{B}, \bigcirc\rangle$ be based on an infinite complete atomic BA. If $\mathfrak{R}$ has uncountably many atoms, then we use Proposition 7.6. Otherwise, $F_{c}$ is free and generated by a chain, by Lemma 7.3 . Hence $F_{c} \notin$ Ult , because no free ultrafilter in a complete BA is generated by a chain (see, e.g., [21, lemma 43]). But Gpt $\subseteq$ Ult, by Lemma 6.8. Thus, we have $\mathrm{F}_{\mathrm{c}} \notin$ Gpt. 
In consequence, there are BCAs that do not satisfy $\left(\mathrm{pF}_{\mathrm{c}}\right)$. In Theorem 7.9 we will construct a certain class of BCAs satisfying $\left(\mathrm{pF}_{\mathrm{c}}\right)$. Moreover, in Theorem 10.1 we will show that elements of the class are also GCAs. Now we will focus on some properties of infinite atomic BCAs in which $\mathrm{F}_{\mathrm{c}}$ is a G-point.

THEOREM 7.8. Let $\mathfrak{R}$ be an infinite atomic $B C A$ satisfying $\left(\mathrm{pF}_{\mathrm{c}}\right)$. Then:

1. $\mathfrak{R}$ has countably many atoms.

2. For all $x, y \in R: x \subset y$ iff either $x \circ y$ or both $\mathrm{At}_{x}$ and $\mathrm{At}_{y}$ are infinite.

3. $\mathrm{F}_{\mathrm{c}}$ is the only $G$-point of $\Re$ being a free filter.

4. $\mathbf{G p t}=\mathbf{P F}_{\mathrm{At}} \cup\left\{\mathrm{F}_{\mathrm{c}}\right\}$ and $\mathbf{G p t}$ is countable.

5. $\mathrm{F}_{\mathrm{c}} \in \mathbf{U l t}$ iff Gpt $\subseteq$ Ult iff $\Re$ is a $B C A^{\circ}$.

6. If $\mathrm{F}_{\mathrm{c}} \in \mathbf{U l t}$, then $\mathbf{G p t}=\mathbf{U l t}$ and $\Re$ is incomplete and finite-cofinite.

Proof. Ad 1. Directly from Proposition 7.6.

$A d 2$. The " $\Rightarrow$ "-part we obtain directly from Lemma 4.3. For the " $\Leftarrow$ "-part assume that $\mathrm{At}_{x}$ and $\mathrm{At}_{y}$ are infinite. Then, by Lemma 7.1, for any $z \in \mathrm{F}_{\mathrm{c}}$ we have: $z \circ x$ and $z \circ y$. Hence, since $\mathrm{F}_{\mathrm{c}} \in \mathbf{G p t}$, by condition ( $\mathrm{r} 3$ ), we have $x \mathrm{C} y$.

Ad 3. By Lemma 7.5, $\mathrm{F}_{\mathrm{c}}$ is included in any free filter. In consequence, if $\mathrm{F}_{\mathrm{c}}$ belongs to Gpt, then its maximality in the set of G-points excludes any free filter from the set Gpt . In other words, none of the other G-points is a free filter.

$A d$ 4. By our assumption and Proposition 6.3, we have $\mathbf{P F}_{\mathrm{At}} \cup\left\{\mathbf{F}_{\mathrm{c}}\right\} \subseteq \mathbf{G p t}$. In virtue of point 2, since $\Re$ is atomic, all G-points different from $F_{c}$ belong to $\mathbf{P F} \mathbf{F}_{\mathrm{At}}$. Thus, also $\mathbf{G p t} \subseteq \mathbf{P F}_{\mathrm{At}} \cup\left\{\mathrm{F}_{\mathrm{c}}\right\}$. So, by point 1, Gpt is countable.

Ad 5. It is obvious that $\mathbf{P F}_{\mathrm{At}} \subseteq$ Ult. So if $\mathrm{F}_{\mathrm{c}} \in$ Ult then Gpt $\subseteq$ Ult, by point 3 . Moreover, if Gpt $\subseteq$ Ult then $\mathrm{F}_{\mathrm{c}} \in \mathbf{U}$ Utt, also by point 3. Hence $\Re$ is a $\mathrm{BCA}^{\circ}$, by Corollary 7.2. Finally, if $\mathfrak{R}$ is a $\mathrm{BCA}^{\circ}$ then $\mathbf{G p t} \subseteq$ Ult, by Lemma 6.8. So $\mathrm{F}_{\mathrm{c}} \in \mathbf{U l t}$, by our assumption.

Ad 6. Suppose that $\mathrm{F}_{\mathrm{c}} \in \mathbf{U l t}$. Then $\mathbf{G p t} \subseteq$ Ult, by point 5. For the converse inclusion assume that $U \in \mathbf{U l t}$. But each ultrafilter in a given $\mathrm{BA}$ either belongs to $\mathbf{P F}_{\mathrm{At}}$ or is free. In the first case, $U \in \mathbf{G p t}$, by point 3. In the second case, by Lemma 7.5, we have $\mathrm{F}_{\mathrm{c}} \subseteq U$. So $U=\mathrm{F}_{\mathrm{c}} \in \mathbf{G p t}$. Thus, we also obtain that $\mathbf{U l t} \subseteq \mathbf{G p t}$.

Finally, since $\mathrm{F}_{\mathrm{c}} \in \mathbf{G p t} \cap \mathbf{U l t}$, by Proposition 6.2(1), $\mathfrak{R}$ is incomplete (we can also use point 5 and Proposition 7.7). Furthermore, $\mathfrak{R}$ is finite-cofinite, by Corollary 7.2. $\square$

Below we describe a construction of BCAs which satisfy $\left(\mathrm{pF}_{\mathrm{c}}\right)$. The construction is a generalization of model 6.19 from [14].

THEOREM 7.9. Let $\mathfrak{B}$ be an infinite atomic BA with countably many atoms. For all $x, y \in R$ we put:

$$
x \subset y: \Longleftrightarrow \text { either } x \circ y \text { or both } \mathrm{At}_{x} \text { and } \mathrm{At}_{y} \text { are infinite. }
$$

Then:

1. For all $x, y \in R$ we have:

$$
x \ll y \Longleftrightarrow\left(x \leq y \wedge A t_{x} \text { is finite }\right) \vee\left(x \leq y \wedge A t_{-y} \text { is finite }\right) .
$$

2. $\langle\mathfrak{B}, \mathrm{C}\rangle$ is a $B C A$.

7 This point was suggested by one of the reviewers. Obviously, in the case when $F_{c} \in \mathbf{U l t}$, by point 5 , we obtain that $\mathrm{C}=\mathrm{O}$. 
3. For the chain $\mathrm{C}_{\mathrm{c}}$ from Lemma 7.3 and $x \in R$ we have:

$$
x \text { overlaps with each member of } \mathrm{C}_{\mathrm{c}} \Longleftrightarrow A t_{x} \text { is infinite. }
$$

4. $\mathrm{C}_{\mathrm{c}}$ is a pre-point and $\mathrm{F}_{\mathrm{c}}$ is a G-point of $\langle\mathfrak{B}, \mathrm{C}\rangle$.

Proof. Ad 1. For all $x, y \in R: x \ll y$ iff $x \varnothing-y$ iff $x \perp-y$ and either $\mathrm{At}_{x}$ or At $_{-y}$ is finite iff either both $x \leq y$ and $\mathrm{At}_{x}$ is finite, or both $x \leq y$ and $\mathrm{At}_{-y}$ is finite.

Ad 2. It is obvious that $\langle\mathfrak{B}, \mathrm{C}\rangle$ satisfies $(\mathrm{C} 0)-(\mathrm{C} 2)$ and $(\mathrm{C} 4)$. Moreover, from point 1 we have $\left(\exists_{\ll}\right)$. For $(\mathrm{C} 3)$ suppose that $x \leq y$ and $z \mathrm{C} x$. If $z \circ x$, then $z \circ y$. If both $\mathrm{At}_{z}$ and $\mathrm{At}_{x}$ are infinite, then $\mathrm{At}_{y}$ is also infinite; and so $z \mathrm{C} y$.

Ad 3. Condition $(\dagger)$ is obvious.

Ad 4. Let At $:=\left\{a_{1}, a_{2}, \ldots\right\}$. For any $x \in \mathrm{C}_{\mathrm{c}}$ the set $\mathrm{At}_{-x}$ is finite, since for some $n \in \omega$ we have $-x=a_{0} \sqcup \cdots \sqcup a_{n}$. For (r1) suppose that $u, v \in \mathrm{C}_{\mathrm{c}}$ and $u \neq v$. Then $u \leq v$ or $v \leq u$. Thus $u \ll v$ or $v \ll u$, by point 1 . For (r2) notice that in the case $u \in \mathrm{C}_{\mathrm{c}}$ for some $n \in \omega$ we have: $v:=-a_{1} \sqcap \cdots \sqcap-a_{n} \sqcap-a_{n+1} \leq-a_{1} \sqcap \cdots \sqcap-a_{n}=u$. So $v \in \mathrm{C}_{\mathrm{c}}$ and $v \ll u$, by point 1 . For (r3) suppose that $x$ and $y$ overlap with each element of $\mathrm{C}_{\mathrm{c}}$. Then, by $(\dagger), \mathrm{At}_{x}$ and $\mathrm{At}_{y}$ are infinite. So $x \mathrm{C} y$.

To round off the proof we use Lemma 7.3.

Thus, directly from Theorems 7.8 and 7.9 we obtain the following characterization of infinite atomic BCAs having the Fréchet filter as a G-point:

Corollary 7.10. Let $\langle\mathfrak{B}, \mathrm{C}\rangle$ be an infinite atomic $B C A$. Then $\mathrm{F}_{\mathrm{c}} \in \mathbf{G p t}$ if and only if $\mathfrak{B}$ has countably many atoms and for all $x, y \in R$ :

$$
x \mathrm{C} y \Longleftrightarrow \text { either } x \circ y \text { or both } \mathrm{At}_{x} \text { and } \mathrm{At}_{y} \text { are infinite. }
$$

ReMARK 7.11. (i) For any set $X$, let $\mathrm{P}(X)$ be the power set algebra of $X$. Any given atomic $\mathrm{BA}$ is monomorphic to $\mathrm{P}(\mathrm{At})$. Namely, it is isomorphic to the field of sets $\left\{\mathrm{At}_{x} \mid x \in R\right\}$. If a $\mathrm{BA}$ is complete, it is isomorphic to $\mathrm{P}(\mathrm{At})$. Thus, any atomic $\mathrm{BA}$ with countably infinitely many atoms is isomorphic to a dense subalgebra of $\mathrm{P}(\omega)$. Moreover, if such a BA is complete, then it is isomorphic to $\mathrm{P}(\omega)$.

(ii) With reference to Theorems 7.8 and 7.9, let us observe that we can distinguish three kinds of infinite atomic BAs with countably many atoms:

1. finite-cofinite-they are incomplete and isomorphic to the finite-cofinite algebra of sets on $\omega$ (shortly: FC $(\omega)$ );

2. complete - they are isomorphic to $\mathrm{P}(\omega)$;

3. incomplete, but not finite-cofinite - each is isomorphic to a dense subalgebra of $\mathrm{P}(\omega)$. This type includes, for example, the Borel algebra of the space of rational numbers with the standard topology (see, e.g., [22, p. 21]).

If in such BAs we introduce the relation $C$ from Theorem 7.9, we obtain BCAs in which $\mathrm{F}_{\mathrm{c}}$ is a G-point. For the first type of BAs, we get $\mathrm{C}=0, \mathrm{~F}_{\mathrm{c}} \in \mathbf{U l t}$ and Gpt $=\mathbf{U l t}$; but for the other two types, $\bigcirc \subsetneq \mathrm{C}$ and $\mathrm{F}_{\mathrm{c}} \notin \mathbf{U l t}$ (cf. Proposition 4.4 and Theorem 7.8).

By Theorems 7.8 and 7.9, the above three types of BAs constitute all infinite atomic BAs satisfying $\left(\mathrm{pF}_{\mathrm{c}}\right)$.

It is often walking on thin ice when trying to infer philosophical conclusions from mathematical results. Nevertheless, we venture to sketch a spatial perspective on Corollary 7.10. The notion of Fréchet filter is one of the central concepts of the theory of atomic Boolean algebras. As is well-known, there are algebras whose Stone spaces of 
points contain the filter, and in the case a BA has the Fréchet filter, it is a subset of every point (ultrafilter) of the Stone space. The notion of a Grzegorczyk point is motivated by geometrical intuitions about the pre-theoretical concept of point. Thus, the sentence "the Fréchet filter is a Grzegorczyk point" might be viewed as saying that the Fréchet filter of a BA satisfies the geometrical intuitions about points. Corollary 7.10 shows that the class of algebras whose Fréchet filter meets those intuitions is relatively narrow, and as such, it testifies to a kind of limitation result about the dependency between the spatial, region-based motivations and purely algebraic constructions. Most of the Boolean algebras have Fréchet filters that do not follow the idea of a point embodied in the definition of Grzegorczyk's.

§8. Grzegorczyk contact algebras. We extend the axioms for the class of BQCAs with the following second-order postulates:

$$
\begin{gathered}
\forall_{x, y \in R}\left(x \circ y \Longrightarrow \exists_{Q \in \mathbf{Q}} \exists_{u \in Q} u \leq x \sqcap y\right), \\
\forall_{x, y \in R}\left(x \subset y \wedge x \perp y \Longrightarrow \exists_{Q \in \mathbf{Q}} \forall_{u \in Q}(u \circ x \wedge u \circ y)\right),
\end{gathered}
$$

called Grzegorczyk axioms, introduced in [16, Proposition 6.1] and equivalent to the single axiom from [19]. Every BQCA which satisfies $\left(\mathrm{G}_{\circ}\right)$ and $\left(\mathrm{G}_{\perp}\right)$ is called a Grzegorczyk contact algebra (GCA in short).

It has been demonstrated in [16, Theorem 6.4$]$ that $(\mathrm{C} 0)-(\mathrm{C} 3)$ together with $\left(\mathrm{G}_{0}\right)$ and $\left(\mathrm{G}_{\perp}\right)$ entail $(\mathrm{C} 4)$ and $\left(\mathrm{C} 3_{\mathrm{c}}\right){ }^{8}$ All other conditions from Lemma 4.1, i.e., (C5), $\left(\exists_{\ll}\right)$, (ext $\left.)_{\leq}\right)$and $\left(\right.$ext $\left._{C}\right)$, hold as well. (We also get $\left(\exists_{\ll}\right)$ from the reflexivity of $\bigcirc$ on $R^{+},\left(\mathrm{G}_{\circ}\right),(\mathrm{r} 2)$ and (3.7).) Therefore,

Proposition 8.1. Every GCA is a BCA.

Notice that in virtue of Lemma 5.1, we have that:

LeMma 8.2. All atomic BCAs satisfy $\left(\mathrm{G}_{\circ}\right)$.

All GCAs with $C=\bigcirc$ will be called GCAs ${ }^{\circ}$. In this case we get $\left(G_{\perp}\right)$ in a trivial way. Thus, we obtain:

Proposition 8.3. Every atomic $B C A^{\circ}$ is a $G C A^{\circ}$ and vice versa.

Thanks to Proposition 6.9 we obtain examples of $\mathrm{GCAs}^{\circ}$ in which Gpt $\subsetneq$ M.Rnd (all infinite complete atomic $\left.\mathrm{BCAs}^{\circ}\right){ }^{9}$

In any GCA, by $\left(G_{\circ}\right)$, the definition of Gpt and the reflexivity of $\bigcirc$ on $R^{+}$, we have:

$$
\forall_{x \in R^{+}} \exists_{\mathfrak{p} \in \mathbf{G p t}} x \in \mathfrak{p} .
$$

The following properties of G-points of $\mathfrak{R}$ play an important role in the analysis of GCAs (see [14, 18]):

$$
\forall x, y \in R\left(x \circ y \Longleftrightarrow \exists_{\mathfrak{p} \in \mathbf{G p t}}(x \in \mathfrak{p} \wedge y \in \mathfrak{p})\right),
$$

8 The first proof that (C4) holds for Grzegorczyk structures can be found in [3], proposition 4.1, yet for a system with a different set of axioms. For a detailed comparison see [17].

9 See also model 6.15 from [14] and other models given in $[16,18]$ in which we have Gpt $\subsetneq$ M.Rnd and which are atomless. 


$$
\begin{aligned}
& \forall_{x, y \in R}\left(x \subset y \Longleftrightarrow \exists_{\mathfrak{p} \in \mathbf{G p t}} \forall_{z \in \mathfrak{p}}(z \circ x \wedge z \circ y)\right), \\
& \forall_{x, y \in R}\left(x \ll y \Longleftrightarrow \forall_{\mathfrak{p} \in \mathbf{G p t}}\left(y \in \mathfrak{p} \vee \exists_{z \in \mathfrak{p}} z \perp x\right)\right), \\
& \forall_{x, y \in R}\left(x \ll y \Longleftrightarrow \forall_{\mathfrak{p} \in \mathbf{G p t}}(y \in \mathfrak{p} \vee-x \in \mathfrak{p})\right) .
\end{aligned}
$$

We get that all points of a given GCA are ultrafilters if and only if it is trivial (and so each of its region is separated from its complement):

THEOREM 8.4. In any GCA:

Gpt $\subseteq$ Ult iff $\forall_{x \in R} x \varnothing-x$ iff $\ll$ is reflexive iff $\mathrm{C}=0$ iff $\mathfrak{R}$ is $a G C A^{\circ}$.

Proof. From $(\mathrm{df} \ll)$ and (8.4) we obtain:

$$
\forall_{x \in R} x \boldsymbol{\ell}-x \text { iff } \ll \text { is reflexive iff } \forall_{\mathfrak{p} \in \mathbf{G p t}} \forall_{x \in R}(x \in \mathfrak{p} \vee-x \in \mathfrak{p}) .
$$

So we use (3.8), Lemma 6.8 and a property of ultrafilters.

§9. G-points, ultrafilters, principal filters and atoms of GCAs. Due to the seminal duality established by Stone [24] for BAs, ultrafilters are candidates for points of GCAs and therefore a question how they relate to G-points comes to mind in a natural way. A partial answer, formulated in [14, 18], boils down to the following theorem which is closely related to Corollary 6.10.(1). The proof of the theorem refers to two nontrivial properties of BAs: any infinite $B A$ has a free ultrafilter and an infinite antichain and no free ultrafilter in a complete $B A$ is generated by a chain (for the latter see, e.g., [21, Lemma 43]).

THEOREM 9.1. If $\Re$ is a complete GCA, the following conditions are equivalent:
a. $\mathfrak{R}$ is finite,
b. Gpt is finite,
c. Ult $\subseteq$ Gpt,
d. Ult $=$ Gpt.

In Remark 7.11, we showed that $\langle\mathrm{FC}(\omega), 0\rangle$ is an example of an infinite incomplete atomic $\mathrm{BCA}^{\circ}$ (and so also $\mathrm{GCA}^{\circ}$ ) in which we have $\mathbf{G p t}=\mathbf{U l t}$.

In the sequel, we will consider the relationship between atoms and principal filters in GCAs. To begin with, we show that we can strengthen Proposition 6.3.

Lemma 9.2. For any $x \in R: x \in$ At iff $\uparrow x \in$ M.Rnd.

Proof. The " $\Rightarrow$ "-part we obtain directly from Propositions 6.3 and 8.1. For the “ $\Leftarrow$ "-part, assume that $x \notin$ At. Then for some $y \in R^{+}$we have $y \lessgtr x$. Moreover, by (8.1), for some $\mathfrak{p}_{0} \in \mathbf{G p t}$ we have $y \in \mathfrak{p}_{0}$. Hence $\uparrow x \subsetneq \mathfrak{p}_{0}$. Therefore, $\uparrow x$ is not maximal in the family of all round filters, since $\mathfrak{p}_{0} \in$ Rnd. Thus, we have $\uparrow x \notin$ M.Rnd.

Furthermore, we obtain the following theorem which previously appeared in $[14,18]$ and which is a strengthening of Proposition 6.3 and Corollary 6.4 for GCAs:

Theorem 9.3. In any GCA, for any $x \in R: x \in$ At iff $\uparrow x \in$ Gpt iff $\uparrow x \in$ Ult.

Proof. Obviously: $x \in$ At iff $\uparrow x \in$ Ult. From Propositions 6.3 and 8.1 for any $x \in$ At we have $\uparrow x \in \mathbf{G p t}$. So if $\uparrow x \in$ Ult then $\uparrow x \in \mathbf{G p t}$. Let $\uparrow x \in \mathbf{G p t}$. Then $\uparrow x \in \mathbf{M}$.Rnd, by Proposition 6.1. Hence $x \in$ At, by Lemma 9.2. So also $\uparrow x \in$ Ult. 
From the above theorem we have the following generalization of Proposition 6.6:

Corollary 9.4. In any $G C A, \mathbf{P F} \cap \mathbf{U I t}=\mathbf{P F}_{\mathrm{At}}=\mathbf{G p t} \cap \mathbf{P F}$.

\$10. G-points and free filters in infinite atomic GCAs. This section is devoted to GCAs based on an infinite atomic BAs. In this case condition $\left(\mathrm{pF}_{\mathrm{c}}\right)$ is independent from the axioms of GCAs.

Firstly, there are GCAs that do not satisfy $\left(\mathrm{pF}_{\mathrm{c}}\right)$. Indeed, in the light of Proposition 8.1 and Propositions 7.6 and 7.7, respectively, we get that: no atomic GCA with uncountably many atoms or complete atomic $G C A^{\circ}$ with infinitely many atoms fulfills $\left(\mathrm{pF}_{\mathrm{c}}\right)$.

Secondly, we show that there are GCAs satisfying $\left(\mathrm{pF}_{\mathrm{c}}\right)$. They are given by the construction we used in Theorem 7.9.

THEOREM 10.1. Let $\mathfrak{B}$ be an infinite atomic BA with countably many atoms. For all $x, y \in R$ we put:

$x \mathrm{C} y: \Longleftrightarrow$ either $x \circ y$ or both $\mathrm{At}_{x}$ and $\mathrm{At}_{y}$ are infinite.

Then $\langle\mathfrak{B}, \mathbb{C}\rangle$ satisfies conditions $1-4$ from Theorem 7.9 and moreover:

5. $\langle\mathfrak{B}, \mathrm{C}\rangle$ is a $G C A$ satisfying $\left(\mathrm{pF}_{\mathrm{c}}\right)$.

Proof. From point 2 and Lemma 8.2 we have that $\langle\mathfrak{B}, C\rangle$ satisfies $\left(\mathrm{G}_{\circ}\right)$. For $\left(\mathrm{G}_{\perp}\right)$, if $x \mathrm{C} y$ and $x \perp y$, then $\mathrm{At}_{x}$ and $\mathrm{At}_{y}$ are infinite, by point 2 and Lemma 4.3. Now we use $(\dagger)$.

Finally, notice that in any infinite atomic GCAs in which $\left(\mathrm{pF}_{\mathrm{c}}\right)$ holds, the filter $\mathrm{F}_{\mathrm{c}}$ has all properties from Theorem 7.8 .

§11. Topological spaces of G-points for infinite atomic GCAs. This section shows how the relation between G-points and ultrafilters influences the topological spaces of G-points.

Let $\mathfrak{R}=\langle\mathfrak{B}, C\rangle$ be a GCA. For any region $x$ of $\mathfrak{R}$ we put Ult $(x):=\{U \in \mathbf{U l t} \mid$ $x \in U\}$. It is well-known that the family $\{\operatorname{UIt}(x) \mid x \in R\}$ is a topological basis on Ult. By the Stone space of $\mathfrak{B}$ we mean - in the standard way - the topological space $\mathcal{S}(\mathfrak{B})$ in which points are ultrafilters of $\mathfrak{B}$ and the topology is introduced via the basis $\{\operatorname{Ult}(x) \mid x \in R\} .^{10}$

Furthermore, for any region $x$ of $\mathfrak{R}$ we define the set of all its internal G-points:

$$
\operatorname{Irl}(x):=\{\mathfrak{p} \in \mathbf{G p t} \mid x \in \mathfrak{p}\} .
$$

Of course, $\operatorname{Irl}(0)=\emptyset$ and $\operatorname{Irl}(1)=\mathbf{G p t}$. Moreover, for all $x, y \in R$ : if $x \circ y$, then $\operatorname{Irl}(x \sqcap y)=\mathbf{I r l}(x) \cap \operatorname{Irl}(y) ; x \leq y$ iff $\mathbf{I r l}(x) \subseteq \operatorname{Irl}(y)$ (see [18, p. 826]). It is routine to verify that the family $\mathscr{B}_{\mathfrak{R}}:=\{\operatorname{Irl}(x) \mid x \in R\}$ is a topological basis on Gpt. Let $\mathcal{T}_{\mathfrak{R}}:=\left\langle\mathbf{G p t}, \mathscr{O}_{\mathfrak{R}}\right\rangle$ be the topological space introduced via $\mathscr{B}_{\mathfrak{R}}$. One can prove that every set from $\mathscr{B}_{\mathfrak{R}}$ is regular open in $\mathcal{T}_{\mathfrak{R}}$, and so $\mathcal{T}_{\mathfrak{R}}$ is semi-regular. Moreover, $\mathcal{T}_{\mathfrak{R}}$ is concentric, i.e., it is a $\mathrm{T}_{1}$-space and each point $\mathfrak{p} \in \mathbf{G p t}$ has a local basis $\mathscr{B}_{\mathfrak{R}}^{\mathfrak{p}}$ satisfying the following

10 For topological spaces and Stone spaces see, e.g., [2, 13], [22, chap. 3] and [1], respectively. 
condition:

$$
\forall_{U, V \in \mathscr{B}_{\mathfrak{R}}^{\mathfrak{p}}}(U=V \vee \mathrm{Cl} U \subseteq V \vee \mathrm{Cl} V \subseteq U)
$$

It is known that every topological space having the same properties as $\mathcal{T}_{\mathfrak{R}}$ is also a regular space (see $[14,16,18]$ for details). ${ }^{11}$

In [18, p. 839] it is shown that: the base $\mathscr{B}_{\mathfrak{R}}$ consists of clopen sets if and only if $\mathfrak{R}$ is a $G C A^{\circ}$. Thus, in the general case, the base of $\mathcal{T}_{\mathfrak{R}}$ does not have to consist of clopen sets. However, below in Proposition 11.3 we can prove that for any infinite atomic GCA $\mathfrak{R}$ satisfying $\left(\mathrm{pF}_{\mathrm{c}}\right), \mathcal{T}_{\mathfrak{R}}$ is a continuous image of a certain Boolean space (i.e., a Hausdorff, compact and zero-dimensional space).

Given a topological space $\mathcal{T}=\langle S, \mathscr{O}\rangle$, its compactification is a pair $\langle\mathcal{K}, e\rangle$ such that: $\mathcal{K}=\langle K, \mathscr{K}\rangle$ is a compact space, $e: S \rightarrow K$ is an embedding (an injective continuous map) of $\mathcal{T}$ into $\mathcal{K}$ (so $e$ is a homeomorphism between $S$ and $e[S]$ ) and the set $e[S]$ is dense in $\mathcal{K}$. Moreover, we say that $\mathcal{K}$ is a compactification of $\mathcal{T}$ iff $e$ is the identity map.

The following lemma will be used in the proof of the last theorem of the paper.

Lemma 11.1. Let $\mathcal{T}_{1}=\left\langle S_{1}, \mathscr{O}_{1}\right\rangle, \mathcal{T}_{2}=\left\langle S_{2}, \mathscr{O}_{2}\right\rangle$ and $\mathcal{K}=\langle K, \mathscr{K}\rangle$ be topological spaces such that:

- $\langle\mathcal{K}, e\rangle$ is a compactification of $\mathcal{T}_{1}$ for some $e: S_{1} \rightarrow K$,

- $e^{\prime}: S_{1} \rightarrow S_{2}$ is an embedding of $\mathcal{T}_{1}$ into $\mathcal{T}_{2}$,

- $f: K \rightarrow S_{2}$ is a continuous surjection such that for any $p \in S_{1}, f(e(p))=e^{\prime}(p)$.

Then $\left\langle\mathcal{T}_{2}, e^{\prime}\right\rangle$ is a compactification of $\mathcal{T}_{1}$.

Proof. $\mathcal{T}_{2}$ is compact as a continuous image of the compact space $\mathcal{K}$. Therefore, it remains to show that $e^{\prime}\left[S_{1}\right]$ is dense in $\mathcal{T}_{2}$. Let $p \in S_{2} \backslash e^{\prime}\left[S_{1}\right], V \in \mathscr{O}_{2}$ and $p \in V$. Thus, for some $k \in K \backslash e\left[S_{1}\right]$ we have $f(k)=p$ and $f^{-1}[V] \in \mathscr{K}$. So, $f^{-1}[V] \cap e\left[S_{1}\right] \neq \emptyset$ and $\emptyset \neq f\left[f^{-1}[V] \cap e\left[S_{1}\right]\right] \subseteq f\left[f^{-1}[V]\right] \cap f\left[e\left[S_{1}\right]\right] \subseteq V \cap e^{\prime}\left[S_{1}\right]$.

The following proposition is standard:

Proposition 11.2. If $\mathfrak{R}$ is infinite and atomic, then $\mathcal{S}(\mathfrak{B})$ is (unique up to homeomorphism) the Stone-Cech compactification of $\left\langle\mathbf{P F}_{\mathrm{At}}, \wp\left(\mathbf{P F}_{\mathrm{At}}\right)\right\rangle$.

Now we prove:

Proposition 11.3. Let $\mathfrak{R}=\langle B, C\rangle$ be an infinite atomic GCA satisfying $\left(\mathrm{pF}_{\mathrm{c}}\right)$. Then $\mathcal{T}_{\mathfrak{R}}$ is a continuous image of $\mathcal{S}(\mathfrak{B})$ under the function $\mathrm{f}: \mathbf{U l t} \rightarrow \mathbf{G p t}\left(=\mathbf{P F}_{\mathrm{At}} \cup\left\{\mathrm{F}_{\mathrm{c}}\right\}\right)$ defined via:

$$
\mathrm{f}(U):= \begin{cases}U & \text { if } U \in \mathbf{P F}_{\mathrm{At}}, \\ \mathrm{F}_{\mathrm{c}} & \text { if } U \text { is free. }\end{cases}
$$

Furthermore, if $\mathrm{F}_{\mathrm{c}} \in \mathbf{U} \mathbf{l t}$, then $\mathrm{f}$ is the identity map.

11 Lemma A2 in [16] showed that every concentric topological space is regular. Moreover, it is obvious that every concentric space has a linearly ordered base at each its point, i.e., is a lob-spaces (see, e.g., [6, p. 37]). Motivated by one of the reviewers, in Appendix A we prove that all regular lob-spaces are concentric. The result is not directly related to the problems discussed in the paper, yet it is novel and relevant for the theory of GCAs. 
Proof. The proof is trivial if $\mathbf{F}_{\mathrm{c}} \in \mathbf{U l t}$, because then we have $\mathbf{G p t}=\mathbf{U l t}$, and so $\mathrm{f}(U)=U$ for any $U \in$ Ult. In other cases, to show that $\mathrm{f}$ is continuous it is enough to prove that for any $x \in R$, the $\operatorname{set}^{-1}[\operatorname{Irl}(x)]$ is open in $\mathcal{S}_{\mathfrak{R}}$. We consider three cases.

Let $x$ be finite, i.e., $x=a_{1} \sqcup \cdots \sqcup a_{n}$, for some $a_{1}, \ldots, a_{n} \in \operatorname{At}$. Then $\operatorname{Irl}(x)=$ $\left\{\uparrow a_{1}, \ldots, \uparrow a_{n}\right\}=\mathbf{U I t}(x)=\mathrm{f}^{-1}[\mathbf{I r l}(x)]$.

Let $x$ be cofinite, i.e., $x=-\left(a_{1} \sqcup \cdots \sqcup a_{n}\right)$, for some $a_{1}, \ldots, a_{n} \in$ At. Then again we have $\mathrm{f}^{-1}[\operatorname{Irl}(x)]=\mathbf{U l t}(x)$, since for any free ultrafilter $U$ we have: $U \in \mathrm{f}^{-1}[\operatorname{Irl}(x)]$ iff $U \in \mathbf{U l t}(x)$. Indeed, assume that $U \in \mathrm{f}^{-1}[\operatorname{Irl}(x)]$, i.e., for some $\mathfrak{p} \in \operatorname{Irl}(x): \mathfrak{p}=\mathrm{f}(U)$. Then $x \in \mathrm{f}(U)$. Furthermore, $\mathrm{f}(U)=\mathrm{F}_{\mathrm{c}}$, since $U$ is free. So $\mathrm{f}(U) \in \operatorname{Irl}(x)$. The other way round, if $\mathrm{f}(U) \in \operatorname{Irl}(x)$, then $x \in \mathrm{f}(U)=\mathrm{F}_{\mathrm{c}}$, since $U$ is free. Finally, because $\mathrm{F}_{\mathrm{c}} \subseteq U$ (see Lemma 7.5), we have $x \in U$, as required.

Let $x$ be neither finite nor cofinite. Then $\mathrm{F}_{\mathrm{c}} \notin \mathbf{U I t}$ and $\operatorname{Irl}(x)=\left\{\uparrow a \mid a \in \mathrm{At}_{x}\right\}$, because $x \notin \mathrm{F}_{\mathrm{c}}$ (see Lemma 7.5). Therefore, $\mathrm{f}^{-1}[\operatorname{Irl}(x)]=\operatorname{Irl}(x) \subseteq \operatorname{Ult}(x)$. Yet the principal ultrafilters correspond to isolated points of $\mathcal{S}_{\mathfrak{R}}$; and so $\mathrm{f}^{-1}[\operatorname{Irl}(x)]$ must be open in $\mathcal{S}(\mathfrak{B})$.

Thanks to Lemma 11.1 and Propositions 11.2 and 11.3 we have the following theorem which is a stronger version of Theorem 6.33 from [14]:

TheOREM 11.4. Let $\mathfrak{R}=\langle\mathfrak{B}, \mathrm{C}\rangle$ be an infinite atomic $G C A$ satisfying $\left(\mathrm{pF}_{\mathrm{c}}\right)$. Then:

1. $\mathcal{T}_{\mathfrak{R}}$ is compact.

2. $\mathcal{T}_{\mathfrak{R}}$ is the one-point compactification of the discrete space $\left\langle\mathbf{P F}_{\mathrm{At}}, \wp\left(\mathbf{P F}_{\mathrm{At}}\right)\right\rangle$ with $\mathbf{F}_{\mathrm{c}}$ as the "point at infinity."

3. $\mathscr{O}_{\mathfrak{R}}=\wp\left(\mathbf{P F}_{\mathrm{At}}\right) \cup\left\{X \cup\left\{\mathrm{F}_{\mathrm{c}}\right\} \mid X \subseteq \mathbf{P F}_{\mathrm{At}}\right.$ and $\mathbf{P F}_{\mathrm{At}} \backslash X$ is finite $\}$.

4. $\mathcal{T}_{\mathfrak{R}}$ is homeomorphic to the Stone space $\mathcal{S}(\mathrm{FC}(\mathfrak{B}))$ of the subalgebra $\mathrm{FC}(\mathfrak{B})$ of $\mathfrak{B}$ composed of all its finite-cofinite elements. ${ }^{12}$

Proof. Ad 1. In the light of Proposition 11.3, $\mathcal{T}_{\mathfrak{R}}$ is compact as a continuous image of the compact space $\mathcal{S}(\mathfrak{B})$.

Ad 2. Of course, id: $\mathbf{P F}_{\mathrm{At}} \rightarrow \mathbf{G p t}$ is an embedding of $\left\langle\mathbf{P F}_{\mathrm{At}}, \wp\left(\mathbf{P F}_{\mathrm{At}}\right)\right\rangle$ into $\mathcal{T}_{\mathfrak{R}}$. By Proposition 11.2, $\mathcal{S}(\mathfrak{B})$ is a compactification of $\left\langle\mathbf{P F}_{\mathrm{At}}, \wp\left(\mathbf{P F}_{\mathrm{At}}\right)\right\rangle$. By Proposition $11.3, \mathcal{T}_{\mathfrak{R}}$ is a continuous image of $\mathcal{S}(\mathfrak{B})$ under the function $\mathrm{f}:$ Ult $\rightarrow \mathbf{G p t}$. Thus, since $\mathrm{f}$ is the identity map on $\mathbf{P F}_{\mathrm{At}}, \mathcal{T}_{\mathfrak{R}}$ is a compactification of $\left\langle\mathbf{P F}_{\mathrm{At}}, \wp\left(\mathbf{P F}_{\mathrm{At}}\right)\right\rangle$, by Lemma 11.1.

Furthermore, $\mathcal{T}_{\mathfrak{R}}$ has the unique G-point $F_{\mathrm{c}}$ which does not belong to $\mathbf{P F}_{\mathrm{At}}$, and therefore $\mathcal{T}_{\mathfrak{R}}$ must be the one-point compactification of $\left\langle\mathbf{P F}_{\mathrm{At}}, \wp\left(\mathbf{P F}_{\mathrm{At}}\right)\right\rangle$.

Ad 3. Directly from point 2.

$A d 4 . \mathrm{F}_{\mathrm{c}}$ is the only free ultrafilter in $\mathrm{FC}(\mathfrak{B})$, which entails that $\mathcal{S}(\mathrm{FC}(\mathfrak{B}))$ is exactly the one-point compactification of $\left\langle\mathbf{P F}_{\mathrm{At}}, \wp\left(\mathbf{P F}_{\mathrm{At}}\right)\right\rangle$. See also point 3 .

With respect to Remark 7.11(ii), note that in the light of Theorem 11.4(4), there are non-isomorphic GCAs $\mathfrak{R}_{1}$ and $\mathfrak{R}_{2}$ which have homeomorphic spaces $\mathcal{T}_{\mathfrak{R}_{1}}$ and $\mathcal{T}_{\mathfrak{R}_{2}}$, respectively. For example, it is true for $\langle\mathrm{FC}(\omega), 0\rangle$ and $\langle\mathrm{P}(\omega), \mathrm{C}\rangle$, where $\mathrm{C}$ is such as in Theorem 7.9 (see Proposition 8.3 and Theorem 10.1). Namely, in both cases, the dual spaces are homeomorphic to $\mathcal{S}(\mathrm{FC}(\omega))$. This may be a reason to question the usefulness of the theorem. However, the primary objective of representation theorems is to find

12 This fact and Proposition 11.3 are consistent with the well-known fact that $\mathcal{S}(\mathrm{FC}(\mathfrak{B}))$ is a continuous image of $\mathcal{S}(\mathfrak{B})$. 
a familiar set-theoretical construction to which the initial structure can be reduced. Concerning this, the theorem does a good job, so to say. The representation theorems in the spirit of Stone [24], Düntsch \& Winter [11] and Dimov \& Vakarelov [8] were presented by us in our earlier works $[16,18]$ where we produced object duality theorems for the class of Grzegorczyk contact algebras that satisfy an algebraic version of the countable chain condition. ${ }^{13}$ In the context of this paper, we are only interested in the much narrower class of Grzegorczyk contact algebras. The results obtained, including the representation theorem above, broaden the understanding of Grzegorczyk pointfree topology, so far a largely neglected system of a region-based theory of space.

§A. Appendix: concentric topological spaces and lob-spaces. In this appendix, we prove that all regular lob-spaces are concentric, and in consequence, a topological space is concentric if and only if it is a regular lob-space.

Let $\mathcal{T}=\langle S, \mathscr{O}\rangle$ be a topological space. We call $\mathcal{T}$ a lob-space provided that for each $p \in S$ there is a linearly ordered base $\mathscr{B}^{p}$ at $p$ (see, e.g., [6, definition 1.1]), i.e., $\mathscr{B}^{p}$ satisfies the trichotomy condition:

$$
\forall_{U, V \in \mathscr{B}}(U=V \vee U \subsetneq V \vee V \subsetneq U)
$$

Furthermore, $\mathcal{T}$ is concentric iff it is a $\mathrm{T}_{1}$-space and for any $p \in S$ there is a base $\mathscr{B}^{p}$ at $p$ such that:

$$
\forall_{U, V \in \mathscr{B} p}(U=V \vee \mathrm{Cl} U \subseteq V \vee \mathrm{Cl} V \subseteq U)
$$

Obviously, all concentric spaces are lob-spaces. Now we prove that all regular lobspaces are concentric.

THeOREM A.1 A topological space is concentric if and only if it is a regular lob-space.

Proof. For the " $\Rightarrow$ "-part see Lemma A2 in [16].

For the " $\Leftarrow$ "-part suppose that $\mathcal{T}=\langle S, \mathscr{O}\rangle$ is a regular lob-space and $p \in S$. Then $\mathcal{T}$ is a $\mathrm{T}_{1}$-space and there is a base $\mathscr{B}^{p}$ at $p$ satisfying the trichotomy condition and such that for any $B \in \mathscr{B}^{p}$ there is a $U \in \mathscr{O}$ such that $p \in U \subseteq \mathrm{Cl} U \subseteq B$.

For some ordinal $\alpha$, let $\left\langle W_{\beta} \mid \beta<\alpha\right\rangle$ be a coinitial subset of $\left\langle\mathscr{B}^{p}, \subseteq\right\rangle$ such that for all $\beta<\delta<\alpha$ we have $W_{\delta} \subsetneq W_{\beta} \cdot{ }^{14}$ For every $\beta<\alpha$ there is a $\delta$ such that $\beta \leqslant \delta<\alpha$ and $\mathrm{Cl} W_{\delta} \subseteq W_{\beta}$. Indeed, for $W_{\beta}$ there is an open set $U$ such that $p \in U \subseteq \mathrm{Cl} U \subseteq$ $W_{\beta}$. Yet $\left\langle W_{\beta} \mid \beta<\alpha\right\rangle$ is a base at $p$, so there is a $\delta<\alpha$ such that $W_{\delta} \subseteq U$, and in consequence $\mathrm{Cl} W_{\delta} \subseteq W_{\beta}$, so $\beta \leqslant \delta$. Thus, there is a monotone coinitial subsequence of $\left\langle W_{\beta} \mid \beta<\alpha\right\rangle$ that satisfies (C), as required.

Acknowledgements. We would like to thank anonymous referees whose apt and valuable remarks helped us improve the paper.

This research was funded in part by the National Science Center (Poland), grant number 2020/39/B/HS1/00216 "Logico-philosophical foundations of geometry and topology."

13 We have not been able to eliminate it so far.

14 See, e.g., [20], exercise on p. 68 and Counting Theorem on p. 80. 


\section{BIBLIOGRAPHY}

[1] Balcar, B., \& Simon, P. (1989). Chart of topological duality. In Monk, J. D. and Bonnet, R., editors. Handbook on Boolean Algebras, Vol. 3. Amsterdam: Elsevier, pp. $1235-1237$.

[2] — (1989). Appendix on general topology. In Monk, J. D. and Bonnet, R., editors. Handbook on Boolean Algebras, Vol. 3. Amsterdam: Elsevier, pp. 1239-1268.

[3] Biacino, L., \& Gerla, G. (1996). Connection structures: Grzegorczyk's and Whitehead's definitions of point. Notre Dame Journal of Formal Logic, 37(3), 431-439.

[4] Blass, A. (2016). Boolean algebras and free filters generated by chains. MathOverflow (version: 2016.03.15). Available from: http://mathoverflow.net/q/220041.

[5] Cohn, A. G., Bennett, B., Gooday, J. and Gotts, N. M. (1997). Qualitative spatial representation and reasoning with the region connection calculus. GeoInformatica, $\mathbf{1}(3), 275-316$.

[6] Davis, S. W. (1978). Spaces with linearly ordered local bases. Topology Proceedings 3, 37-51. Available from: http://topology.nipissingu.ca/tp/reprints/ v03/tp03102.pdf.

[7] De Vries, H. (1962). Compact Spaces and Compactifications. Amsterdam: Van Gorcum.

[8] Dimov, G., \& Vakarelov, D. (2006). Contact algebras and region-based theory of space: A proximity approach-I. Fundamenta Informaticae, 74(1), 209-249.

[9] - (2006). Contact algebras and region-based theory of space: A proximity approach-II. Fundamenta Informaticae, 74(2-3), 251-282.

[10] Düntsch, I., \& Winter, M. (2004). Construction of Boolean contact algebras. AI Communications, 17, 235-246.

[11] - (2005). A representation theorem for Boolean contact algebras. Theoretical Computer Science, 347(3), 498-512.

[12] —, (2006). Weak contact structures. In MacCaull, W., Winter, M., and Düntsch, I., editors. Relational Methods in Computer Science. Berlin and Heidelberg: Springer, pp. 73-82.

[13] Engelking, R. (1977). General Topology. Warszawa: PWN.

[14] Gruszczyński, R. (2016). Niestandardowe Teorie Przestrzeni (Non-Standard Theories of Space; in Polish). Toruń: Nicolaus Copernicus University Scientific Publishing House.

[15] Gruszczyński, R., \& Pietruszczak, A. (2009). Space, points and mereology. On foundations of point-free Euclidean geometry. Logic and Logical Philosophy, 18(2), $145-188$.

[16] - (2018). A study in Grzegorczyk point-free topology. Part I: Separation and Grzegorczyk structures. Studia Logica, 106(6), 1197-1238.

[17] , (2018). A comparison of two systems of point-free topology. Bulletin of the Section of Logic, 47(3), 187-200.

[18] — (2019). A study in Grzegorczyk point-free topology. Part II: Spaces of points. Studia Logica, 107(4), 809-843.

[19] Grzegorczyk, A. (1960). Axiomatizability of geometry without points. Synthese, 12(2-3), 228-235.

[20] Halmos, P. (1974). Naive Set Theory. New York: Springer. 
[21] Hamkins, J., \& Seabold, D. (2012). Well-founded Boolean ultrapowers as large cardinal embeddings. Available from: http://arxiv.org/abs/1206.6075.

[22] Koppelberg, S. (1989). General theory of Boolean algebras. In Monk, J. D. and Bonnet, R., editors. Handbook on Boolean Algebras, Vol. 1. Amsterdam: NorthHolland.

[23] Stell, J. G. (2000). Boolean connection algebras: A new approach to the regionconnection calculus. Artificial Intelligence, 122(1-2), 111-136.

[24] Stone, M. (1936). The theory of representation of Boolean algebras. Transactions of the American Mathematical Society, 40(1), 37-111.

[25] Tarski, A. (1956). Foundations of the geometry of solids. In J. Corcoran, editor. Logic, Semantics, Metamathematics. Papers from 1923 to 1938. Oxford: Oxford University Press, pp. 24-29.

[26] Whitehead, A. N. (1929). Process and Reality. New York: MacMillan.

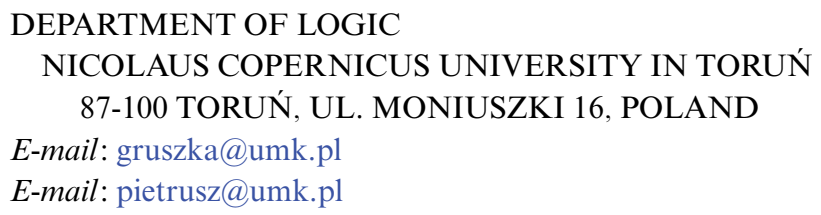

